\title{
Indications for living donor liver transplantation in patients with hepatocellular carcinoma
}

\author{
Yasuhiko Sugawara, Yukihiro Inomata \\ Department of Transplantation/Pediatric Surgery, Postgraduate School of Life Science, Kumamoto University, Kumamoto, Japan \\ Correspondence to: Yasuhiko Sugawara, MD. Department of Transplantation/Pediatric Surgery, Postgraduate School of Life Science, Kumamoto \\ University, 1-1-1 Honjo, Chuo-ku, Kumamoto, Japan. Email: yasusuga-tky@umin.ac.jp.
}

\begin{abstract}
Liver transplantation is the optimal radical therapy for patients with cirrhosis and hepatocellular carcinoma (HCC). The Milan criteria are widely applied for deceased donor liver transplantation (DDLT) in the western countries. Living donor liver transplantation (LDLT), however, prevails in Asian countries due to the extreme shortage of deceased donor organs. In contrast to DDLT, the feasibility of LDLT is not restricted by the national allocation system, and therefore the indications for LDLT in patients with HCC depend on institutional policies that consider both the operative risk to the donor and the survival benefit for the recipient. The results of a nationwide survey as well as the experiences of individual centers demonstrate similar outcomes for patients whose tumors fall within the Milan criteria and those whose tumors extend beyond the Milan criteria.
\end{abstract}

Keywords: Liver transplantation; living donor; hepatocellular carcinoma (HCC)

Submitted Jul 17, 2016. Accepted for publication Aug 04, 2016.

doi: 10.21037/hbsn.2016.09.01

View this article at: http://dx.doi.org/10.21037/hbsn.2016.09.01

\section{Introduction}

Liver transplantation is an established therapeutic option for patients with hepatocellular carcinoma (HCC) with decompensated cirrhosis (1). Liver transplantation is advantageous in that not only the tumor but the underlying disease of the liver are treated simultaneously (2). The efficacy of liver transplantation is maximized when the HCC tumors are limited in number and size (3).

Mazzaferro et al. (4) suggested restricting liver transplantation to only those patients with early $\mathrm{HCC}-$ defined as a single tumor $\leq 5 \mathrm{~cm}$ or up to three tumors $\leq 3 \mathrm{~cm}$ in diameter in the absence of major vessel invasion or extrahepatic tumor spread based on imaging studies. Mazzaferro et al. (4) demonstrated an overall 4-year survival of $75 \%$ and a recurrence-free survival of $83 \%$ with application of these criteria. Many centers worldwide have adopted these criteria to identify suitable liver transplantation candidates from among patients with HCC. Nonetheless, the criteria have been criticized as being too strict, preventing some potential recipients from a second chance at life (5).
The pressure to expand the criteria to allow more patients to benefit from transplantation has led to several proposed schemas (6). Like the Milan criteria, however, most of these newly proposed schemas were designed for application to deceased donor liver transplantation (DDLT). Living donor liver transplantation (LDLT) can only be performed at the strong request of a voluntary donor who has an established relationship with the recipient. In LDLT, the potential graft is dedicated to a specific recipient, and therefore the indications LDLT for patients with HCC may be appropriately expanded.

\section{Asian perspective of LDLT for HCC}

In Asian countries, unlike in western countries, the majority of liver transplantation cases undergo LDLT (7). Liver grafts used in LDLT are not considered a public resource and, therefore, they not limited by the organ allocation system $(7,8)$. In the LDLT setting, the overall survival chance of the recipient as well as the preferences of the donor must be carefully considered. Most LDLT centers in 
Asia now apply some expanded selection criteria for LDLT in patients with HCC (9-14).

The criteria are based on both the number and size of the HCC lesions. The Tokyo group selection criteria for recipients of LDLT for HCC are up to five nodules $\leq 5 \mathrm{~cm}$ in diameter (9). In their series, the expanded selection criteria did not worsen the prognosis of the HCC patients after transplantation compared with that of patients who met the Milan criteria. In 2008, Lee and colleagues (10) proposed the Asan criteria, i.e., up to five nodules $\leq 6 \mathrm{~cm}$ in diameter. The overall and recurrence-free survivals were reported be comparable to those of patients meeting the Milan criteria. Both the Asan and Tokyo criteria only slightly extend the Milan criteria. Like the criteria outlined by the University of California in San Francisco (15) (a single lesion with a diameter of $\leq 6.5 \mathrm{~cm}, 2-3$ tumors $\leq 4.5 \mathrm{~cm}$ in diameter with a total diameter of $8 \mathrm{~cm}$, or up to seven lesions $\leq 7 \mathrm{~cm}$ in diameter), the Asan and Tokyo criteria were developed to achieve results similar to the Milan criteria (6).

Some other centers in Asia have proposed further expansion of the criteria for LDLT in HCC using tumor markers $(11-13,16)$. The Kyoto group (11) proposed newly expanded LDLT criteria, adding the serum des-gammacarboxy prothrombin (DCP) level to the selection criteria, while increasing the upper limit of the number of tumors to ten. These criteria were defined as ten or fewer tumors $\leq 5 \mathrm{~cm}$ in diameter and DPC levels $\leq 400 \mathrm{mAU} / \mathrm{mL}$. The Kyushu group removed the tumor number limitation from their criteria (13). All HCCs $\leq 5 \mathrm{~cm}$ and a serum DCP level $\leq 300 \mathrm{mAU} / \mathrm{mL}$ were included in their selection criteria. The 5-year recurrence rate for patients whose tumors met the Kyushu criteria and exceeded the Milan criteria was $20 \%$, whereas for patients whose tumors met the Milan criteria, the 5 -year recurrence rate was $4 \%$.

The Hangzhou criteria (16) are total tumor diameter $\leq 8 \mathrm{~cm}$ or total tumor diameter $>8 \mathrm{~cm}$, with histopathologic grade I or II and alpha-fetoprotein (AFP) levels $\leq 400 \mathrm{ng} / \mathrm{mL}$. The 5 -year survival rate of the HCC patients whose tumors fulfilled the Hangzhou criteria was $72 \%$. A recent review (17) of 6,012 HCC patients from the China liver transplant registry reported that the Hangzhou criteria expanded the indications for LDLT by $52 \%$ compared with the Milan criteria without increasing the recurrence rate. The Samsung group in Seoul (12) added AFP levels to expand their selection criteria. They advocate LDLT for HCC patients with up to seven tumors $\leq 6 \mathrm{~cm}$ in diameter and a serum AFP level $\leq 1,000 \mathrm{ng} / \mathrm{mL}$. The 5 -year recurrence rate was $16 \%$ for patients who met these criteria. Both the Kyushu and Samsung criteria may be acceptable for LDLT, although the post-transplant HCC recurrence rates are higher than the expected when the Milan criteria are applied.

\section{Limitation of tumor status in LDLT}

Although living grafts are provided to the patients through an established, closed donor-recipient relationship, the indications should be cautiously considered in the context of LDLT as there is a significant risk of donor morbidity. Several studies report that the associated risks of death and severe complications for a donor are as high as $0.3 \%$ and $2 \%$, respectively (18-20), leading to a significant decline in LDLT in the western transplant community (1). LDLT should be performed only at centers of excellence in liver resection and transplantation.

The minimal estimated survival acceptable in LDLT is difficult to define. It is critically important to decide how broadly the LDLT criteria may be appropriately expanded for HCC. The acceptable target outcome may vary from $50 \%$ to $60 \%$ survival at 5 years post-transplant to somewhere closer to that achieved when applying the Milan criteria (21). Maintaining an appropriate balance between recipient benefit and donor risk is crucial (22). Although a recent worldwide survey (18) reported that technical experience does not affect the incidence of living donor morbidity or mortality, major complication rates for living donors continue to be reduced by meticulous and innovative surgical techniques, increased experience-based expertise, and careful donor selection in high-volume LDLT centers (8). In Asian countries, LDLT is widely applied for HCC because of crucial shortage of deceased donor organs (23-25). Some patients with HCC tumors and cirrhosis have few therapeutic options other than LDLT. It may be difficult for the physicians to deny the request for LDLT if a well-informed donor wishes to provide the only potentially curative treatment available to the patient (3). Therefore, a post-transplant, long-term expected survival of $50 \%$ to $60 \%$ may be considered acceptable, especially in Asia.

Clearly, the size and number of HCC tumors are not sufficient for predicting HCC recurrence after transplantation. In addition to biomarkers such as AFP and DCP, fluorine-18-flurodeoxyglucose positron emission tomography (PET) positivity may play a role in predicting HCC recurrence (26). Lee et al. (21) observed excellent post-transplant survival for advanced HCC patients with 
negative PET results despite portal vein tumor thrombosis ( $88 \%$ at 3 years) when serum AFP levels were $\leq 200 \mathrm{ng} / \mathrm{mL}$. The paradigm for selecting patients with HCC for liver transplantation appears to be shifting from morphologic criteria to a combination of biologic, histologic, and morphologic criteria. Tumor biomarkers could contribute to overcome the restrictions of the conventional selection criteria, thereby increasing the number of the patients indicated for LDLT (27). One recent study (28) demonstrated a comparable 5 -year recurrence-free survival rate between LDLT recipients whose tumors exceeded the Milan criteria but who had negative PET and recipients whose tumors met the Milan criteria (81\% vs. $86 \%$ ).

\section{Conclusions}

LDLT still accounts for the majority of liver transplantation cases in many countries. As the graft tissue from living donors is not a public resource, not only the HCC recurrence risk but also the survival chance of the recipient and the wishes of the donor must be taken into account for LDLT candidate selection. Therefore, an expected survival of greater than $50 \%$ to $60 \%$ may be considered acceptable in the LDLT setting. The criteria applied for selection of recipients of LDLT for HCC can be expanded based on the combination of biologic, histologic, and morphologic tumor parameters. Additional studies are needed to before a universal consensus can be reached regarding the application of biomarkers as indicators for LDLT in patients with HCC.

\section{Acknowledgements}

None.

\section{Footnote}

Conflicts of Interest: The authors have no conflicts of interest to declare.

\section{References}

1. Befeler AS, Hayashi PH, Di Bisceglie AM. Liver transplantation for hepatocellular carcinoma. Gastroenterology 2005;128:1752-64.

2. Bruix J, Sherman M, Practice Guidelines Committee, et al. Management of hepatocellular carcinoma. Hepatology 2005;42:1208-36.
3. Tamura S, Sugawara Y, Kokudo N. Section 4. Further expanding the criteria for $\mathrm{HCC}$ in living donor liver transplantation: the Tokyo University experience. Transplantation 2014;97 Suppl 8:S17-20.

4. Mazzaferro V, Regalia E, Doci R, et al. Liver transplantation for the treatment of small hepatocellular carcinomas in patients with cirrhosis. N Engl J Med 1996;334:693-9.

5. Mancuso A, Perricone G. Hepatocellular Carcinoma and Liver Transplantation: State of the Art. J Clin Transl Hepatol 2014;2:176-81.

6. Mazzaferro V, Llovet JM, Miceli R, et al. Predicting survival after liver transplantation in patients with hepatocellular carcinoma beyond the Milan criteria: a retrospective, exploratory analysis. Lancet Oncol 2009;10:35-43.

7. Akamatsu N, Sugawara Y, Kokudo N. Living donor liver transplantation for patients with hepatocellular carcinoma. Liver Cancer 2014;3:108-18.

8. Song GW, Lee SG. Living donor liver transplantation. Curr Opin Organ Transplant 2014;19:217-22.

9. Sugawara Y, Tamura S, Makuuchi M. Living donor liver transplantation for hepatocellular carcinoma: Tokyo University series. Dig Dis 2007;25:310-2.

10. Lee SG, Hwang S, Moon DB, et al. Expanded indication criteria of living donor liver transplantation for hepatocellular carcinoma at one large-volume center. Liver Transpl 2008;14:935-45.

11. Takada Y, Ito T, Ueda M, et al. Living donor liver transplantation for patients with HCC exceeding the Milan criteria: a proposal of expanded criteria. Dig Dis 2007;25:299-302.

12. Kim JM, Kwon CH, Joh JW, et al. Expanded criteria for liver transplantation in patients with hepatocellular carcinoma. Transplant Proc 2014;46:726-9.

13. Shirabe K, Taketomi A, Morita K, et al. Comparative evaluation of expanded criteria for patients with hepatocellular carcinoma beyond the Milan criteria undergoing living-related donor liver transplantation. Clin Transplant 2011;25:E491-8.

14. Ng KK, Lo CM, Chan SC, et al. Liver transplantation for hepatocellular carcinoma: the Hong Kong experience. J Hepatobiliary Pancreat Sci 2010;17:548-54.

15. Yao FY, Ferrell L, Bass NM, et al. Liver transplantation for hepatocellular carcinoma: expansion of the tumor size limits does not adversely impact survival. Hepatology 2001;33:1394-403.

16. Zheng SS, Xu X, Wu J, et al. Liver transplantation 
for hepatocellular carcinoma: Hangzhou experiences.

Transplantation 2008;85:1726-32.

17. $\mathrm{Xu} X, \mathrm{Lu} \mathrm{D}$, Ling $\mathrm{Q}$, et al. Liver transplantation for hepatocellular carcinoma beyond the Milan criteria. Gut 2016;65:1035-41.

18. Cheah YL, Simpson MA, Pomposelli JJ, et al. Incidence of death and potentially life-threatening near-miss events in living donor hepatic lobectomy: a world-wide survey. Liver Transpl 2013;19:499-506.

19. Ghobrial RM, Freise CE, Trotter JF, et al. Donor morbidity after living donation for liver transplantation. Gastroenterology 2008;135:468-76.

20. Brown RS Jr. Live donors in liver transplantation. Gastroenterology 2008;134:1802-13.

21. Lee KW, Yi NJ, Suh KS. Section 5. Further expanding the criteria for HCC in living donor liver transplantation: when not to transplant: SNUH experience. Transplantation 2014;97 Suppl 8:S20-3.

22. Kumaran V. Role of liver transplantation for hepatocellular carcinoma. J Clin Exp Hepatol 2014;4:S97-S103.

23. Azzam AZ. Liver transplantation as a management of

Cite this article as: Sugawara Y, Inomata Y. Indications for living donor liver transplantation in patients with hepatocellular carcinoma. HepatoBiliary Surg Nutr 2016;5(5):429-432. doi: 10.21037/hbsn.2016.09.01 hepatocellular carcinoma. World J Hepatol 2015;7:1347-54.

24. Concejero A, Chen CL, Wang CC, et al. Living donor liver transplantation for hepatocellular carcinoma: a single-center experience in Taiwan. Transplantation 2008;85:398-406.

25. Chan SC, Fan ST, Lo CM, et al. A decade of right liver adult-to-adult living donor liver transplantation: the recipient mid-term outcomes. Ann Surg 2008;248:411-9.

26. Lee JH, Cho Y, Kim HY, et al. Serum Tumor Markers Provide Refined Prognostication in Selecting Liver Transplantation Candidate for Hepatocellular Carcinoma Patients Beyond the Milan Criteria. Ann Surg 2016;263:842-50.

27. Lai Q, Lerut JP. Hepatocellular cancer: how to expand safely inclusion criteria for liver transplantation. Curr Opin Organ Transplant 2014;19:229-34.

28. Kornberg A, Küpper B, Tannapfel A, et al. Patients with non-[18 F]fludeoxyglucose-avid advanced hepatocellular carcinoma on clinical staging may achieve long-term recurrence-free survival after liver transplantation. Liver Transpl 2012;18:53-61. 